\title{
Non-spectroscopic Method for Simultaneous Determination of Thickness and Composition via 4D-STEM
}

Katsuaki Nakazawa ${ }^{1}$, Kazutaka Mitsuishi ${ }^{2}$, Shin-ichi Amma ${ }^{3}$ and Teruyasu Mizoguchi ${ }^{1}$

${ }^{1}$ Institute of Industrial Science, University of Tokyo, Meguro-ku, Tokyo, Japan, ${ }^{2}$ National Institute for Materials Science, Tsukuba, Ibaraki, Japan, ${ }^{3}$ AGC Inc., Yokohama, Kanagawa, Japan

The thickness and composition of a specimen are fundamental information for analyzing image, diffraction, and spectrum acquired by transmission electron microscopy (TEM). The thickness and composition maps are mainly obtained by spectroscopy, such as electron energy loss spectroscopy (EELS) or energy dispersive X-ray spectroscopy (EDS). Since these spectroscopies require a large electron dose, they cannot apply to electron-sensitive materials. In crystalline materials, as alternatives to spectroscopy, imaging and diffraction are used to measure the thickness and composition [1,2]. On the other hand, in non-crystalline materials, there are few alternatives to spectroscopy. Here, we focus on the diffraction patterns obtained by a pixelated detector. The thickness affects the number of scattering events, and the composition affects the scattering cross section. Thus, the diffraction pattern must contain the information of thickness and composition. Furthermore, by grace of the fast pixelated STEM detector, we can acquire the diffraction patterns at all scanning points (four-dimensional scanning TEM (4D-STEM)) [3]. We attempted to make the thickness and composition maps simultaneously from the diffraction patterns acquired by 4D-STEM. We picked up the thickness and composition from experimentally obtained diffraction patterns by comparing simulated diffraction patterns at various thicknesses and compositions. In the comparison, we used the radial distribution function (RDF) of the diffraction pattern because the diffraction pattern of non-crystalline material does not depend on the azimuthal angle.

We selected the 27.0BaO-73.0 $\mathrm{SiO}_{2}(\mathrm{~mol} \%)$ glass as a sample. This sample is known to separate into two phases (Si-pure phase and Ba-rich phases). Figure 1 shows the detailed procedure of our method to determine the thickness and composition. We measured radial distribution functions at all scanning points. The measured RDF at each scanning point is compared with simulated RDFs. We simulated RDFs in the range of the thickness from $0 \mathrm{~nm}$ to $1000 \mathrm{~nm}$ in increments of $1 \mathrm{~nm}$ and in the range of the thickness from $0.0 \mathrm{BaO}-100 \mathrm{SiO}_{2}$ to $100.0 \mathrm{BaO}-0.0 \mathrm{SiO}_{2}$ in $1.0 \mathrm{BaO}$ increments. In the comparison, we calculated the mean squared error (MSE) between experimentally obtained RDF and simulated RDF. We adopted the thickness and composition that simulated the RDF which had the lowest MSE as the thickness and composition at the scanning point. Figure 2(a) and (b) show the thickness and composition maps measured by HAADF and EELS. Thickness map shows the gradual increase from the bottom to the top of the image. The composition map shows the circular low-BaO regions. The heterogeneity of composition attribute to the phase separation. We measured the thickness and composition of the same region by 4D-STEM. Figure 2(c) and (d) show the results. The thickness map also shows gradual increase from the bottom to the top. The error between thickness measured by 4D-STEM and one measured by EELS is less than $10 \%$. The composition map also shows the circular low-BaO regions. The composition in the map ranges from $0.0 \mathrm{BaO}-100.0 \mathrm{SiO}_{2}$ to $43.0 \mathrm{BaO}-57.0 \mathrm{SiO}_{2}$. This is identical to the composition expected from the phase diagram. The 4D-STEM method requires only one-tenth electron dose of the EELS method. In the presentation, we will present the detailed procedure of the 4D-STEM method and pros and cons of the 4D-STEM method. 

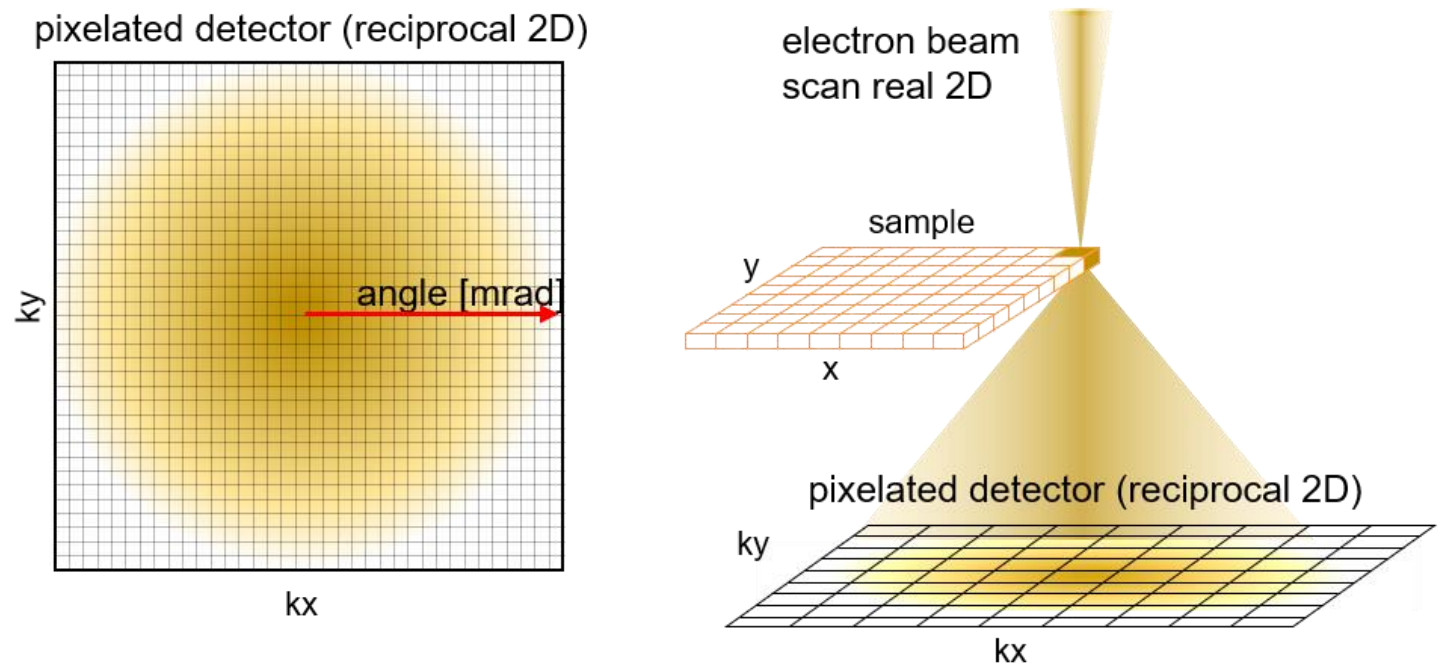

Compare RDF measured by 4D-STEM with simulated RDFs.
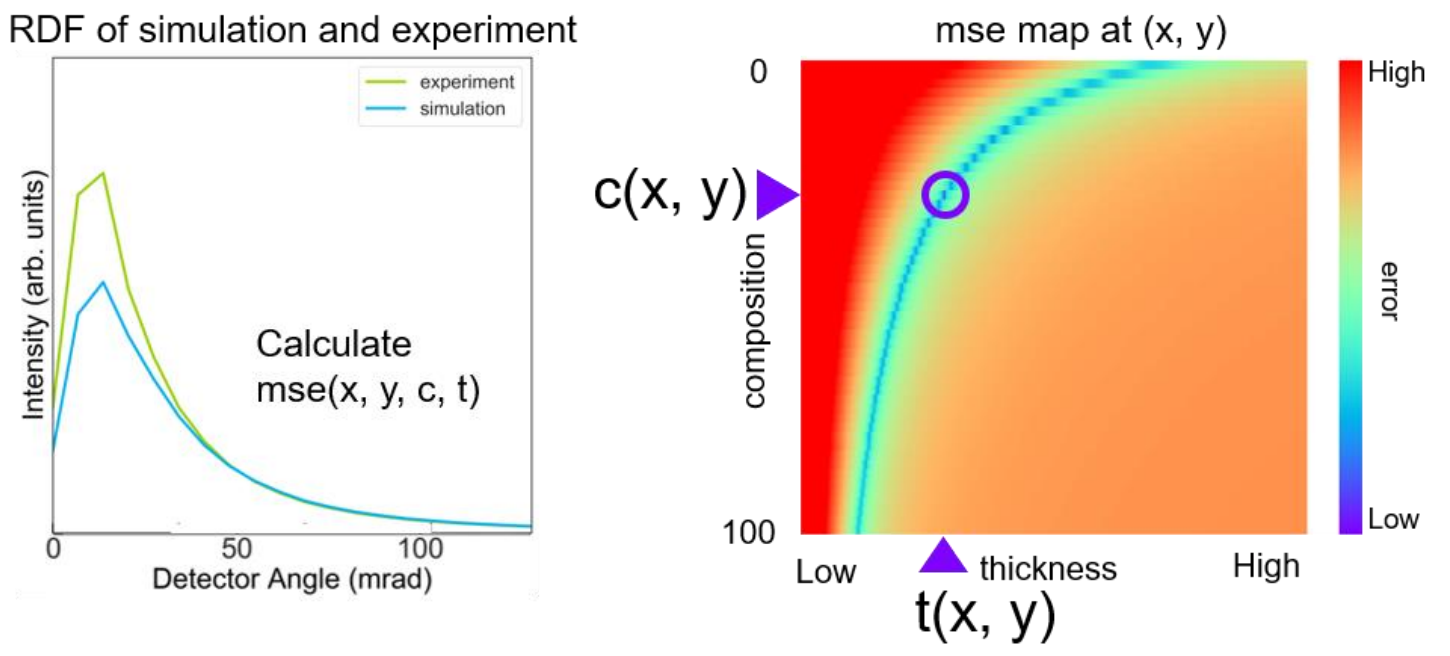

Adopt the thickness and composition that simulate the RDF which has the lowest mse as the $c(x, y)$ and $t(x, y)$ at the scanning point $(x, y)$.

Figure 1. Procedure to determine thickness and composition. 


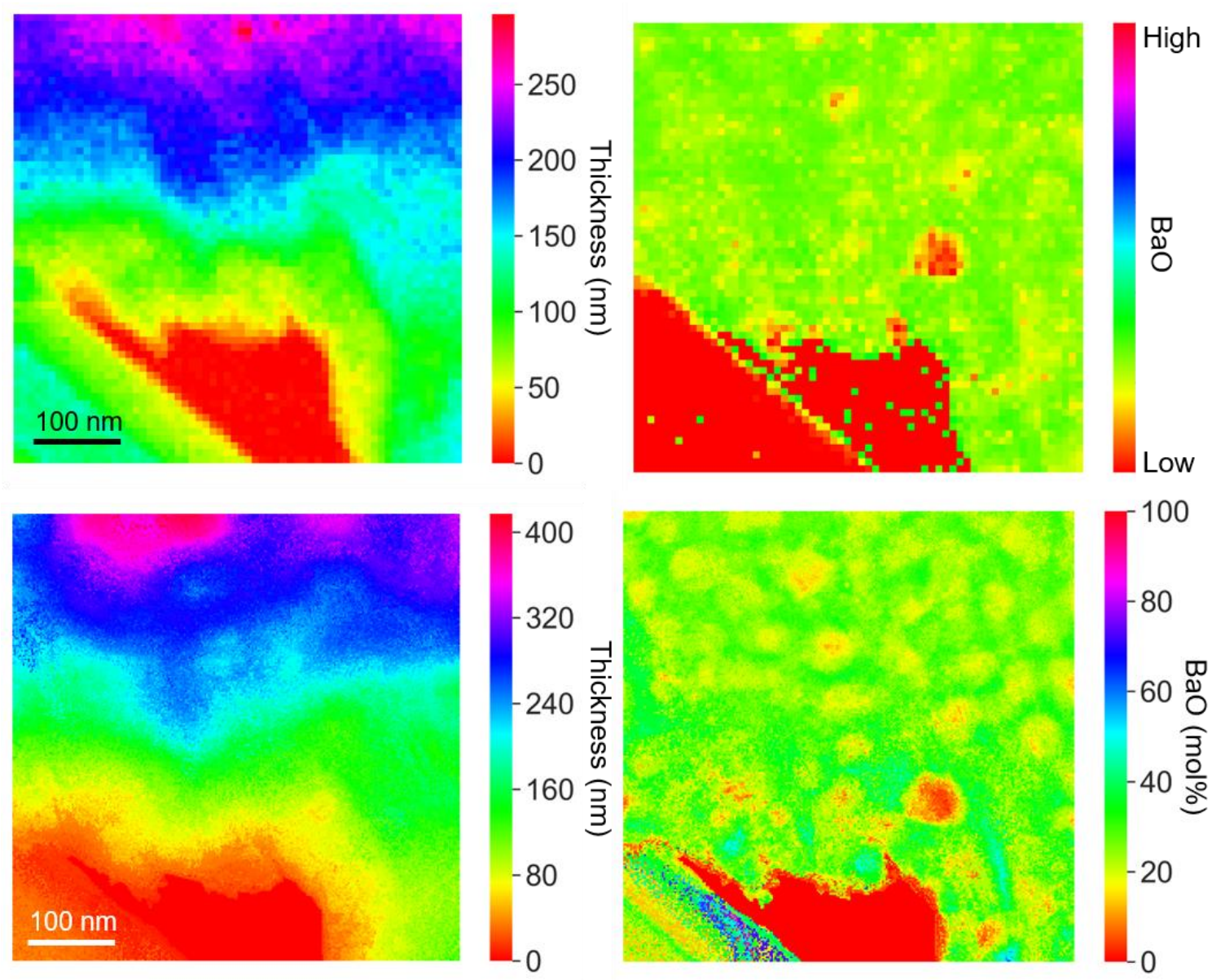

Figure 2. (a) Thickness and (b) composition maps obtained by EELS. (c) Thickness and (d) composition maps obtained by the 4D-STEM method. The bottom left regions of the images are carbon meshes.

\section{References}

[1] R. Ishikawa, A. R. Lupini, S. D. Findlay, and S. J. Pennycook, Microsc. Microanal. 20, 99 (2014).

[2] C. Ophus, P. Ercius, M. Huijben, and J. Ciston, Appl. Phys. Lett. 110, 063102 (2017).

[3] C. Ophus, Microsc. Microanal. 563 (2019). 